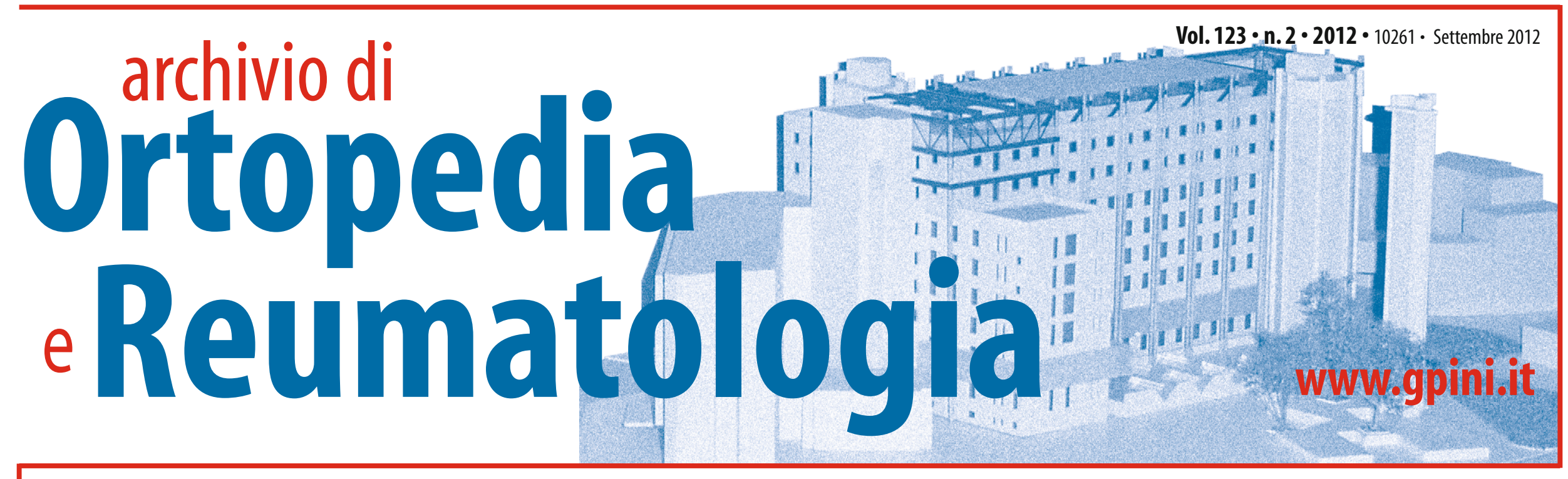

\title{
L'anestesia in ortopedia
}

a cura di Gabriele Cornaggia

\section{Editoriale}

DOI 10.1007/s10261-012-0014-1

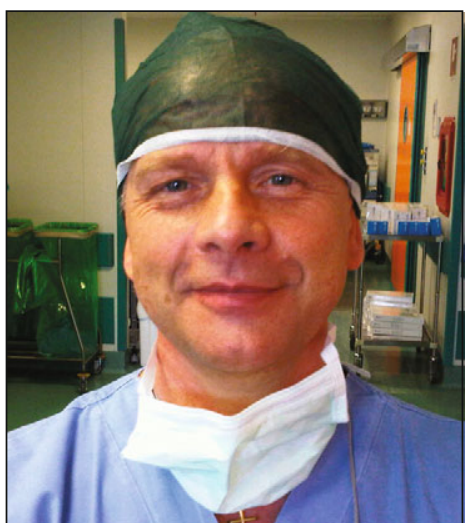

G. Cornaggia

$\Lambda$ d alcuni potrà suonare strano Aleggere un numero della rivista Archivio di ortopedia e reumatologia interamente dedicato all'anestesia. D'altra parte nell'Istituto Ortopedico G. Pini vengono svolti circa 10.000 interventi chirurgici ogni anno il che, considerato il rovescio della medaglia, significa 10.000 anestesie all'anno. Risulta quasi una stonatura, quindi, che solo adesso, per la prima volta da quando viene stampata la rivista, si parli di anestesia. Lortopedia è, tra le discipline chirurgiche, quella che offre all'anestesista le più vaste possibilità per quanto concerne le tecniche anestesiologiche da utilizzare, permettendo di applicare praticamente ogni tipo di anestesia loco-regionale (ALR), associata o meno all'anestesia generale in tutte le sue numerose varianti. Inoltre la chirurgia ortopedica, intesa sia come elezione sia come traumatologia, presenta la più ampia e talvolta complessa tipologia di paziente.

Loccasione si presenta quindi ghiotta; in questo fascicolo abbiamo cercato di trattare gli argomenti di maggior interesse per chi si occupa di anestesia in ortopedia, ma non solo. Abbiamo trattato argomenti come l'anestesia in "day surgery", in pediatria, nel grande anziano e la gestione del sangue, che trovano campi di applicazione anche in altri tipi di chirurgie. Soprattutto abbiamo cercato di focalizzare l'attenzione sugli argo- menti più dibattuti per gli operatori di questo settore e sulle nuove tendenze dell'ALR. Si parlerà molto di ALR. Proprio grazie alle caratteristiche peculiari che la chirurgia ortopedica presenta, il binomio ALR-ortopedia è, nel corso degli anni, diventato di sempre maggiore attualità. Sono stati proprio gli studi clinici eseguiti sull'anestesia in ortopedia quelli che maggiormente hanno contribuito allo sviluppo delle moderne tecniche di anestesia loco-regionale. Infatti è proprio in ortopedia che l'ALR trova la sua massima espressione, offrendo oggi il miglior rapporto rischio-beneficio in senso assoluto. Proprio la particolarità di questa chirurgia, che spesso interessa distretti periferici, ha permesso nel corso degli anni di spostare l'obiettivo da un'anestesia molto invasiva come quella dei blocchi centrali a una sempre più mirata e meno invasiva come quella dei blocchi periferici. Inoltre l'anestesia applicata all'ortopedia è quella che, prima di tutte, ha permesso di evidenziare l'importanza del trattamento di quello che oggi è ritenuto uno dei parametri vitali, cioè il dolore. Infatti proprio il dolore non trattato rappresenta il maggior limite nella ripresa della principale funzione che il sistema osteo-articolare svolge, il movimento. Ma la possibilità di deafferentare la zona dell'intervento offerta dall'utilizzo dell'ALR non offre solo benefici macroscopici come la risoluzione del "sintomo" dolore ma, assicurando una protezione da quello che viene definito lo "stress chirurgico", migliora notevolmente la ripresa funzionale rispetto all'uso della sola anestesia generale [1,2]. Le possibilità offerte dall'ALR in quest'ambito hanno spostato i confini entro i quali aveva lavorato l'anestesista nel passato. L'anestesista oggi non offre solo la possibilità di svolgere l'intervento garantendo il mantenimento delle funzioni vitali, ma interviene nella gestione peri-operatoria in modo attivo. Basti pensare all'importanza che hanno assunto i blocch perinervosi continui in molte procedure riabilitative [1]

Come accennato in precedenza negli ultimi anni l'evoluzione dell'ALR ha spostato l'attenzione da blocchi centrali (spinale, peridu rale) ai blocchi periferici. Se l'a nestesia subaracnoidea presenta caratteristiche che, dopo più di un secolo dalla sua scoperta, la rendono ancora oggi una delle tecniche anestesiologiche più utiliz zate per interventi sull'arto inferiore, l'anestesia epidurale, soprattutto per quanto concerne l'analgesia epidurale continua, pre senta invece, in ambito ortopedico, problematiche che hanno contribuito allo sviluppo dei blocch perinervosi continui. I principal problemi dell'anestesia epidurale derivano dai numerosi effetti collaterali e dalla scarsa maneggevolezza in ambito riabilitativo legati a questa procedura, che spesso portano in secondo piano la sua efficacia antalgica. Inoltre la chirurgia ortopedica è quella che più necessita della profilassi antitromboembolica, sottoponendo il paziente a un rischio superiore al $50 \%$ di sviluppare eventi trombotici in mancanza di terapia. Ed è proprio il rischio emorragico in caso di catetere peridurale e concomitante profilassi antitrom boembolica che ha dato un'ulteriore spinta allo sviluppo delle nuove tecniche di analgesia peri- nervosa. Queste tecniche offrono lo stesso potere antalgico dell'analgesia peridurale, ma con invasività ed effetti collaterali molto inferiori. Il limite principale dei blocchi periferici, se confrontati a quelli centrali, rimane però l'operatore. Spesso la curva di apprendimento è più lenta rispetto a un blocco centrale e la percentuale di successo, anche nel caso di un operatore esperto, difficilmente eguaglia quella di un'anestesia spinale. Lintroduzione delle nuove tecniche di "imaging" sta però migliorando anche quest'aspetto dell'ALR. Da quando Winnie nel 1973 cominciò a descrivere i primi approcci periferici basati sull'utilizzo di larghi volumi di anestetico locale (AL), necessari per poter raggiungere tutte le componenti nervose interessate, è stata fatta molta strada nella direzione opposta. Gli obiettivi attuali sono quelli di migliorare la precisione riducendo volumi e dosi di AL, in modo da aumentare l'efficacia diminuendo al tempo stesso le complicanze. Un grosso aiuto in quest'ottica è stato fornito dall'introduzione dell'elettroneurostimolatore (ENS) prima, e dall'uso degli ultrasuoni in tempi recenti. Entrambe le tecniche permettono di migliorare l'efficacia del blocco riducendo l'"onset" e i dosaggi di AL. Luso dell'ecografia, in particolare, permette la visione diretta delle strutture nervose e dello "spread" dell'AL durante l'iniezio- ne, offrendo ulteriori vantaggi in termini di "onset" ed efficacia del blocco. Anche se l'utilizzo di questa tecnica è strettamente legato all'operatore, la possibilità di visualizzare la diffusione dell'anestetico dovrebbe permettere una maggiore riproducibilità rispetto all'uso dell'ENS. Pochi e discordanti dati si trovano invece in letteratura per quanto riguarda i vantaggi teorici che l'uso dell'ecografia dovrebbe garantire in termini di complicanze.

Anestesia in ortopedia non significa però solo ALR. La chirurgia ortopedica significa anche chirurgia della colonna; un tipo di chirurgia completamente diverso rispetto alla chirurgia degli arti o delle grosse articolazioni che sconfina, talvolta, nella neurochirurgia. Per motivi di spazio questo argomento non verrà trattato nel presente fascicolo, ma vale la pena di ricordare che interventi come la correzione di importanti scoliosi sono la sintesi di tutte le problematiche che l'anestesista può incontrare nella gestione di altri tipi di chirurgia maggiore: ventilazione monopolmonare in caso di toracotomie per stabilizzazioni toraciche, sanguinamenti maggiori, problemi neurologici ed emodinamici dovuti alla sede dell'intervento. L'uso dell'ALR in questo tipo di chirurgia rimane controverso. Linfiltrazione della ferita, per esempio, anche se in alcu-

continua a pag. 2 > 


\section{Board Scientifico}

\section{Direttori Scientifici: M. d'Imporzano, Milano C.Verdoia, Milano Vice Direttori Scientifici: \\ M. Berruto, Milano \\ S. Brambilla, Milano \\ Direttore Responsabile: \\ A. Tropiano, Milano

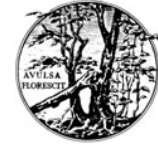

Comitato Scientifico:

P. Bartolozzi, Verona - F.Bertoni, Bologna

F. Biggi, Belluno - R. Capanna, Firenze P. Cherubino, Varese - R.D. D'Ambrosia, New Orleans - L. Del Sasso, Como A. Diméglio, Montpellier - W.F.Enneking, Gainesville - F. Fantini, Milano - P.G. Garbagna, Milano - S. Giannini, Bologna A. Lanzetta, Milano - E. Meani, Milano F.Odella, Milano - A. Parafioriti, Milano G. Randelli, Milano - P. Rossi, Torino - C Verdoia, Milano - V.Zucchi, Milano

\section{Comitato Editoriale:}

S. Caserta, Milano - G. Cornaggia, Milano - C. Corradini, Milano - C. Cucciniello, Milano - F. Di Domenica, Milano - G. Di Luca, Milano - M. Gallazzi, Milano - V. Gerloni, Milano - G. La Maida, Milano - G. Laurà, Milano - F. Maggi, Milano - S. Mapelli, Milano - B. Marelli, Milano - M. Mazza, Milano - E. Paresce, Milano - L. Sinigaglia, Milano - G. Sironi, Milano - F. Torretta, Milano - S. Tosi, Milano A. Ventura, Milano - R. Viganò, Milano

\section{Comitato di Redazione:}

W. Albisetti, Milano - M. Berruto, Milano S.Brambilla, Milano - U.Dacatra,MilanoF.Fischer,Milano - P.L.Gambrioli, Milano L. Pierannunzii, Milano - L. Sinigaglia, Milano - A.Ventura, Milano

Segreteria di Redazione:

N.Zerbi, Milano

\section{Aziende sostenitrici}

Abbott, Campoverde di Aprilia (RM)

Agave Farmaceutici, Prato (PO)

Bayer, Milano

B. Braun, Milano

Ceraver, Bologna

De Puy, Pomezia (RM)

Movi, Milano

Sorin Group Italia, Mirandola (MO)

Synthes, Opera (MI)

Vygon Italia, Padova

\section{Colophon}

\section{Coordinamento editoriale:} Carlotta d'Imporzano

Elena Bernacchi

Journal Department

Springer-Verlag Italia Srl

Via Decembrio 28, 20137 Milano

Tel.:02 5425 9732/65

Fax:025425 9701

e-mail:

carlotta.dimporzano@springer.com

elena.bernacchi@springer.com

\section{Grafica e impaginazione:}

Graphostudio, Milano

Stampa:

Grafiche Erredue, Cirimido (CO)

Registrazione del Tribunale di Milano

N. 400 del 24 ottobre 1981

Registro Nazionale della Stampa

posizione N. 9016

La distribuzione in Italia avvien

ai sensi della legge 675/96

Springer fa parte di

Springer Science + Business Media

springer.com

\section{springer.com}

OSpringer-Verlag Italia, 2012

Via Decembrio 28,

20137 - Milano, Italia

Stampato in Italia

\section{Versione elettronica}

e realizzazione Web:

Anna Gallicchio

smm Srl - Scientific Multimedia

http://www.smm-srl.it

Corso Vercelli 9 - 20144 Milano

e-mail:a.gallicchio@smm-srl.it

\section{(}

ISSN 0390-7368 (versione cartacea)

ISSN 1592-7113 (versione elettronica)

$€ 83,00$ (4 fascicoli)

inclusa la versione on-line

Gli ordini dovranno essere indirizzati a: Springer-Verlag Italia Srl Journal Department

Via Decembrio 28

20137 Milano

e-mail: elena.bernacchi@springer.com

\section{Springer}

la rivista on-line

Vieni a visitarci all'indirizzo

http://www.gpini.it

User ID: arch2001

Password: pini

$\mid \begin{gathered}\text { Di prossima pubblicazione } \\ \text { Biotech in ortopedia: } \\ \text { "Where are we today?" } \\ \text { Curatori: } \\ \text { Walter Albisetti, Massimo Berruto, Alberto Ventura }\end{gathered}$

\section{Erratum}

Archivio di Ortopedia e Reumatologia 2012; 123(1):27-28

DOI 10.1007/s10261-012-0013-2

\section{La correzione chirurgica del piede piatto} giovanile: tecniche a confronto

\section{A. Memeo, A. Acerbi, S. Ronchi, L. Pedretti}

Nella versione originale di questo articolo non è stata indicata la fonte del pannello superiore della Figura 1. Tale immagine è tratta dal seguente articolo:

De Pellegrin M (2007) 15-jährige Erfahrung mit der subtalaren Schrauben-Arthrorise beim kindlichen Plattfuß. Fuß \& Sprunggelenk 5:12-20

Ci scusiamo con l'Autore e con i lettori.

\section{INDICE}

L'ANESTESIA IN ORTOPEDIA

Editioriale a cura di G. Comaggia

I nuovi anticoagulanti orali e l'anestesia in chirurgia ortopedica maggiore: cosa sappiamo? A. Fanelli, D. Ghisi, G. Mensi, B. Pergolotti, G. Danelli

Blocchi perinervosi continui G. Cappelleri

Strategia trasfusionale nella chirurgia ortopedica maggiore P. Ghis

"Day surgery": un modello in continua ascesa A. L. Ambrosoli

Blocco continuo del plesso lombare con approccio posteriore sotto guida ecografica in pazienti sottoposti a chirurgia dell'anca: studio pilota

D. Ghisi, A. Fanelli, M. Gardini, L. La Colla, G. Danelli

Anestesia nel grande anziano: generale vs ALR

L. Pacileo

"Imaging" in anestesia loco-regionale e terapia del dolore: ecografia ed epiduroscopi

\section{A. Somenzi, M. Allegri, P. Grossi}

Dolore cronico: : l ruolo dell'anestesia loco-regionale eco-guidata D. Bugada, C. E. Minella, M. Allegri

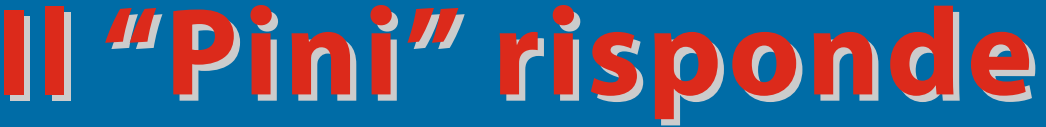

L'Istituto Ortopedico Gaetano Pini sta Si prega di inviare le richieste via e-mail attivando un servizio di linea diretta fra i lettori della rivista e i medici dell'Istituto, che risponderanno a quesiti posti da colleghi su argomenti di carattere specialistico in ambito ortopedico traumatologico, riabilitativo e reumatologico.

al seguente indirizzo:

ilpinirisponde@gpini.it

N.B. Questo servizio è dedicato esclusivamente ai Medici e Chirurghi; non verranno quindi prese in considerazione domande poste da persone che non siano medici.
Editoriale

ni lavori sembra migliorare l'analgesia post-operatoria, non limita in realtà il consumo di oppiacei [3]

Abbiamo parlato del ruolo che l'anestesia oggi riveste anche fuori dalla sala operatoria. In un'ottica moderna di approccio al paziente di tipo multimodale l'anestesia diventa una componente talvolta essenziale del processo te rapeutico. In pratica è necessari cominciare a considerare l'anestesia non solo come un servizio offerto alla chirurgia, ma soprattutto come parte integrante della terapia. Per esemplificare il concetto, basti pensare quanto puo migliorare la ripresa funzionale con un'adeguata condotta anestesiologica dopo un intervento di ortopedia [1] appunto, come de terminate scelte anestesiologiche influenzino addirittura la morta lità dopo grossi interventi di chirurgia addominale [4] o, in ultimo, quale importanza potrebbe avere l'ALR nella recidiva tumorale [5]

Per concludere, ringrazio tutti i miei collaboratori per l'aiuto che ho ricevuto, gli Autori degli articoli per l'ottimo lavoro svolto e in modo particolare G. Cappelleri per la funzione di coordinamento della rivista. Un ringraziamento va anche a tutti gli sponsor che con il loro supporto hanno reso possibile la stampa di questo fascicolo.

G. Cornaggia

Direttore S. C. Anestesia Rianimazione e Terapia del Dolore Istituto Ortopedico G. Pini, Milano

\section{Bibliografia}

Capdevila X, Barthelet Y, Biboulet P et al (1999) Effects of perioperative analgesic technique on the surgical outcome and duration of rehabilitation after major knee surgery. Ane- continua da pag. 1 sthesiology 91:8-15

2. Volk T, Scenk M, Voigt $\mathrm{K}$ et al (2004) Postoperative epidural anesthesia preserves lymphocyte, but non monocyte, immune function after major spine surgery. Anesth Analg 98:10861092

Kjaergaard M, Møiniche S, Olsen KS (2012) Wound infiltration with local anesthetics for post-operative pain relief in lumbar spine surgery: a systematic review. Acta Anaesthesiol Scand 56:282-290

4. Rodgers A, Walker N, Schug S et al (2000) Reduction of postoperative mortality and morbidity with epidural or spinal anaesthesia: results from overview of randomised trials. BMJ 321:1493

Exadaktylos AK, Buggy DJ, Moriarty DC et al (2006) Can anesthetic technique for primary breast cancer surgery affect recurrence or metastasis? Anesthesiology 105:660-664 